\author{
DANIEL GARZA USABIAGA \\ FACULTAD DE FILOSOFÍA Y LETRAS, UNAM
}

\title{
Las Torres de Satélite: ruina de un proyecto que nunca se concluyó
}

MEdio siglo De su Construcción, las Torres de Satélite se encuen-
tran en condiciones de pronunciado deterioro, sufrido a lo largo de
las décadas. No hay duda de que este conjunto escultórico, concebido por el arquitecto Luis Barragán y el escultor Mathias Goeritz en I957, se ha convertido en un espacio representativo del Distrito Federal y su área metropolitana, en un hito y una marca urbana, así como en una pieza emblemática e ilustrativa de la obra de sus autores. Incluso, se puede afirmar que las Torres, durante la segunda mitad del siglo xx, llegaron a constituir, en cierto modo, un símbolo de la modernidad del país entero. ${ }^{\mathrm{I}}$ Debido a todos estos factores -y otros, como una infructuosa controversia sobre los créditos de su autoría-, su historia, lo que les dio su razón de ser, ha quedado prácticamente olvidada, mientras que su análisis crítico se ha postergado eternamente. ${ }^{2}$ Hoy en

I. Esta cuestión la he desarrollado en el texto "Las Torres de Satélite. Escultura moderna y símbolo de modernidad" (de próxima publicación). Ahí explico también que esa obra constituye el primer ejemplo de una segunda forma de entender el arte público en México durante el siglo xx: la escultura monumental urbana que recurre a estructuras primarias y a formas que tienden a la abstracción. Durante la segunda mitad del siglo pasado esta forma de arte público desplazó al muralismo.

2. Un par de publicaciones que han examinado parcialmente las Torres de Satélite son Larissa Pavlioukova y Adrián Soto, "El tema de la torre en la obra de Mathias Goeritz", en Ida Rodríguez Prampolini y Ferruccio Asta (eds.), Los ecos de Mathias Goeritz. Catálogo de la exposición, México, Antiguo Colegio de San Ildefonso/Universidad Nacional Autónoma de México-Instituto de Investigaciones Estéticas, I997, pp. I43-I68, y Louise Noelle, Luis Barragán. Búsqueda y creatividad, México, Universidad Nacional Autónoma de México, 1996, p. I43. Estos dos textos, especializados 


\section{28}

día se olvida que las Torres son un resto, lo que queda de un proyecto urbano que fracasó por completo en el intento de materializar sus intenciones. Uno de los propósitos de este artículo es sacar de nuevo a la luz algunos aspectos del proyecto original que enmarca a las Torres, para así ponderar la enorme distancia que separa al ideal del estado presente de las cosas. En segundo término, se persigue discutir de manera crítica esa obra, especialmente como parte del programa, compartido por sus dos autores, de la arquitectura emocional. Más que llegar a conclusiones finales, se pretende iniciar una reflexión que, en este caso, parte de la restitución de un motivo, el de la torre, que hacia la segunda mitad del siglo xx ya resultaba totalmente anacrónico. ¿¿Significa esto una restauración? Y, de ser así, ¿¿de qué tipo? Éstas son algunas de las cuestiones a las que se busca dar respuesta aquí.

\section{Punto de partida}

Las Torres de Satélite se construyeron en 1957, con el fin de marcar la entrada a una nueva ciudad, del todo autónoma, que se ubicaría fuera de la ciudad de México. Ciudad Satélite fue el nombre de este proyecto dirigido por el arquitecto Mario Pani, quien concibió tal empresa como la primera de una red de ciudades similares alrededor de la gran capital. Pani invitó al arquitecto tapatío Luis Barragán para proyectar una plaza monumental que sirviera como puerta de entrada a esa nueva urbe. A su vez, Barragán llamó a su colaborador Mathias Goeritz, con quien ya había trabajado en proyectos afines, aunque de menor escala, en dos ocasiones. Goeritz, proveniente de Europa, se había establecido en Guadalajara en 1949 y a principios de los años cincuenta Barragán lo conoció y lo invitó a diseñar una escultura para la entrada del fraccionamiento Jardines del Pedregal. Se trata de El animal del Pedregal (I95I), que se convirtió rápidamente en símbolo distintivo de la zona. Los autores hicieron

en el estudio de la obra de cada uno de los autores, son representativos del por lo general somero análisis de las Torres de Satélite. El ensayo que, hasta la fecha, ha estudiado con mayor profundidad las Torres de Satélite es el de Peter Krieger, "Potencial simbólico y actualidad conceptual del arte urbano de Mathias Goeritz", en Paisajes urbanos. Imagen y memoria, México, Universidad Nacional Autónoma de México-Instituto de Investigaciones Estéticas, 2006, pp. 183-227. Mi investigación "Las Torres de Satélite. Escultura moderna y símbolo de modernidad" coincide en varios puntos con su análisis. Sin embargo, ninguno de estos textos ha revisado la construcción de las Torres dentro del contexto más amplio del proyecto de la nueva Ciudad Satélite. 
mancuerna de nueva cuenta en el desarrollo Jardines del Bosque en Guadalajara (1957). Estas colaboraciones, entre otras, son evidencia de una empresa compartida en que los proyectos de ambos autores se identificaban y que se conoce a través de lo que Goeritz publicitó en el Manifiesto de I954 como arquitectura emocional, ejemplificada con su Museo Experimental El Eco (1952). Esta arquitectura se situó de manera crítica ante el estilo internacional que privilegiaba la funcionalidad respecto a cualquier otra consideración. En oposición a ello, el proyecto compartido de Goeritz y Barragán juzgaba que lo más importante en la arquitectura no es la función, sino provocar emoción.

Además de inscribirse en el proyecto de la arquitectura emocional, la discusión de las Torres de Satélite no debe perder de vista su contexto original, es decir, el umbral de una nueva ciudad totalmente moderna. Más que esto, para Pani Ciudad Satélite contaba con un carácter anticipatorio que la hacía, de hecho, una ciudad del futuro. Las intenciones urbanísticas de los proyectos de Pani se remontan a su propuesta relativa al crucero Insurgentes-Reforma, que parte del concurso convocado en 1944 por el periódico Excélsior para realizar un monumento a la madre. Será también en este proyecto donde Pani, junto a José Luis Cuevas, funde el Taller de Planificación y Urbanismo del Banco Internacional Inmobiliario. ${ }^{3}$ Posteriormente, Pani concreta algunas de sus propuestas sobre urbanismo al construirse la Ciudad Universitaria. Sin embargo, no será hasta 1957, en Ciudad Satélite, cuando Pani vea del todo realizadas sus intenciones con el proyecto de construcción de una ciudad creada ex nibilo.

El diseño de Ciudad Satélite, así como el terreno que ocuparía, dependieron del encargo que recibió el Taller de Planificación y Urbanismo, dirigido por Pani, para elaborar el Plan Regional Norte de la ciudad de México, zona donde se impulsaba el crecimiento industrial, especialmente en Naucalpan, Estado de México. El terreno estaba marcado por las líneas ferroviarias que iban hacia el norte. Sería aquí donde se vislumbraría un eje vial —al que se llamó "central”- que conectaría a la capital con Ciudad Satélite y a ésta con la autopista a Querétaro, hasta llegar — después de pasar por Matehuala y Saltillo—a Estados Unidos. El Eje Central era, en sí, parte de la materialización del sueño de la Carretera Panamericana, al que ese país nunca prestó mucha atención, mientras que México celebraba haber sido la primera nación en terminar de construir

3. Mario Pani. Arquitecto, México, Universidad Autónoma Metropolitana-Azcapotzalco/Limusa, I999, pp. 63-7I. 
su parte de la red. ${ }^{4}$ Como se puede apreciar, la ruta de modernización escogida por México en esta época era clara. Se decidió que Ciudad Satélite se ubicara en el kilómetro I4 de ese Eje Central, conforme al ideal de Pani de crear una ciudad fuera de la ciudad. Esta solución también respondía a las políticas sobre desarrollo urbano impuestas por el entonces regente Ernesto Uruchurtu (1952I967), que limitaban el crecimiento de la ciudad en la zona centro y daban pie a la creación de nuevos asentamientos en la periferia. El gobierno del Estado de México aprobó la construcción de Ciudad Satélite el 9 de enero de 1957.5 Las labores de construcción empezaron casi de inmediato.

Meses después, el I2 de septiembre, Pani dictó la conferencia "México. Un problema, una solución” ante la Sociedad de Arquitectos Mexicanos. Ahí presentó su proyecto de Ciudad Satélite, sus características, ventajas y potencial. Esta conferencia se publicó posteriormente en distintas ediciones como una herramienta de promoción tanto de las ideas de Pani como del proyecto de la nueva urbe. ${ }^{6}$ Cabe mencionar que en la edición original se incluyó la primera representación gráfica de las Torres, estructura que para esas fechas ya estaba en proceso de construcción. También se publicó otra serie de ilustraciones, de extraordinaria calidad, que dieron a conocer otros aspectos y espacios de la nueva ciudad: sus pasos a desnivel, las supermanzanas, el centro comercial, entre otros. Tal como se revela en el texto de Pani, lo que en general define la modernidad de Ciudad Satélite es su orden y su organización racionales, su entera planificación para llegar a ser una "ciudad con límites", capaz de satisfacer las demandas de sólo un determinado número de habitantes, en este caso no más de 200000 . La demanda del crecimiento demográfico se iría satisfaciendo, según el ideal de Pani, mediante la construcción de otras ciudades similares que formarían una red de órbitas alrededor de la capital.

La concepción de la nueva Ciudad Satélite parte de las reflexiones de Pani sobre la forma de solucionar los problemas de la creciente concentración urbana establecida, a raíz del centralismo, en la capital. También incluye una revi-

4. Wendy Waters, "Remapping Identities: Road Construction and Nation Building in Postrevolutionary Mexico", en Stephen Lewis y Mary Kay Vaughan (eds.), The Eagle and The Virgin. Nation and Cultural Revolution in Mexico, 1920-1940, Durham, Duke University Press, 2006, pp. 232-235.

5. Gaceta del Gobierno. Órgano del Gobierno Constitucional del Estado de México, Toluca de Lerdo, t. LXXXIV, núm. I3, miércoles I4 de agosto de I957, p. 2.

6. Un caso de esto es el sobretiro del número 60 de la revista Arquitectura-México, diciembre de 1957. 


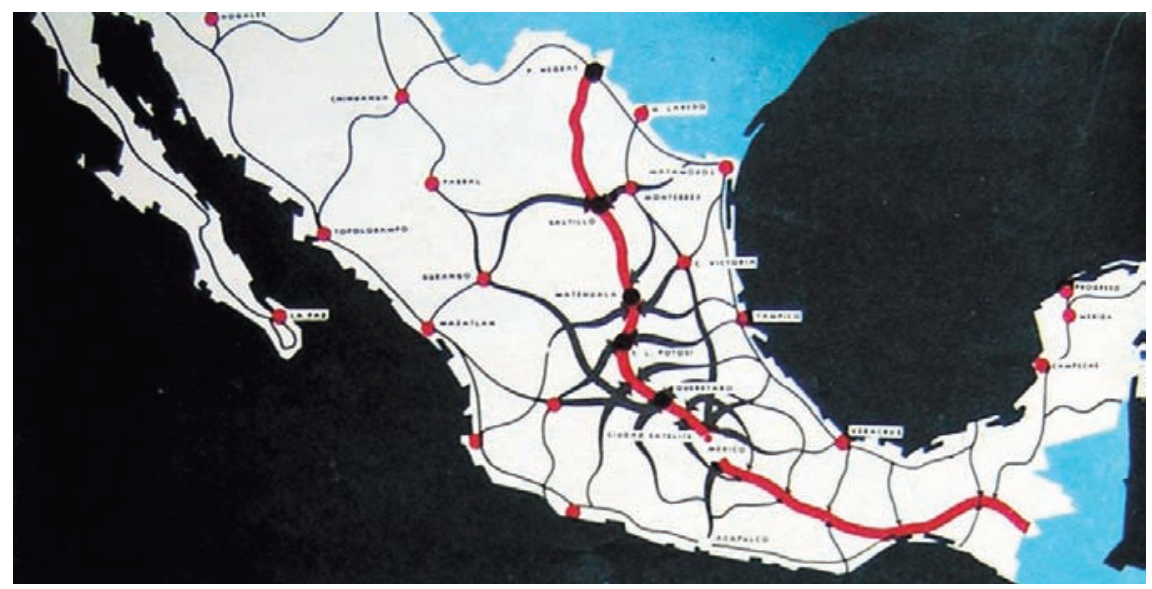

I. Ubicación de Ciudad Satélite en el Eje Central. Imagen tomada de Pani, "México. Un problema, una solución”, Arquitectura-México, núm. 6o, diciembre de 1957, p. 40. Fondo Mathias Goeritz. Archivo del Centro Nacional de Investigación, Documentación e Información de Artes Plásticas del Instituto Nacional de Bellas Artes.

sión histórica de distintas propuestas de urbanismo y una particular perspectiva del ser humano que Pani imagina como usuario de su proyecto. ${ }^{7}$ Sobre esto último, Pani juzga necesario crear espacios de reposo y recreación para que el hombre pueda descansar, pensar y reflexionar como "la única forma civilizada y culta de entender y de vivir la vida humana" ${ }^{8}$ Este tipo de espacios, donde figurarían las áreas verdes, son "vitales" para el autor del proyecto. Como el término lo sugiere, hay una clara influencia de las ideas de José Ortega y Gasset en la concepción del "usuario imaginario" propia de los planes de Pani. El factor de la convivencia como pilar del espacio social y punto de partida para la creación de una sociedad es una constante inquietud de ese arquitecto, que resuena de acuerdo con las ideas del autor de La rebelión de las masas. ${ }^{9}$ Las previsiones

7. Un par de análisis que estudian y exponen este último tema son el de Russell Ellis y Dana Cuff (eds.), Architects' People, Oxford University Press, 1989, y el de Simon Richards, Le Corbusier and the Concept of the Self, New Haven, Yale University Press, 2003.

8. Mario Pani, "México. Un problema, una solución", texto de la conferencia que el autor presentó el I2 de septiembre de 1957 en la tribuna de la Sociedad de Arquitectos Mexicanos, p. 5. Publicación incluida en Arquitectura-México, núm. 60, op. cit.

9. José Ortega y Gasset, La rebelión de las masas, Madrid, Alianza, I979 (Ia. ed., Revista de Occidente, 1930). 
que persiguen el propósito social "de fomentar la convivencia sin segregaciones" en los proyectos de Pani son una constante que se aprecia, por ejemplo, en las unidades habitacionales conocidas como multifamiliares. ${ }^{\text {IO }}$ Es un hecho que, como Ortega y Gasset, Pani veía un proceso civilizatorio como fruto de la convivencia social, antagónica a la disociación que sólo produce barbarie. ${ }^{\text {II }}$ Más aún, Pani y Ortega y Gasset coinciden en ver la ciudad moderna como la representación de la existencia actual; Pani, de hecho, llama "hombres-urbe" a sus contemporáneos. ${ }^{\mathrm{I2}}$ Sin embargo, va más allá de esto y, de acuerdo con el filósofo español, anuncia que el destino, la victoria o la derrota del hombre se encuentra no en el ejercicio de su libertad y su vitalidad, sino en el devenir de la urbe. Aquí, Pani suena a Le Corbusier, sobre todo por el tono de urgencia que imprime a sus palabras en el último capítulo de Vers une Architecture. ${ }^{\mathrm{I3}}$

En cuanto a su análisis de propuestas en la historia del urbanismo, Pani critica los proyectos que se proponen reconstruir la ciudad dentro de sus límites - como el Plan de Haussmann para París o el Plan Voisin (I925) de Le Corbusier-, ya que poseen un carácter destructivo que se dirige a la historia de la fábrica urbana. Peor aún, estas propuestas funcionarían, con el tiempo, tan sólo como un paliativo respecto a los problemas más graves de la época. En cambio, de manera evidente, las propuestas que Pani prefiere son las que plantean ciudades autónomas alrededor de un centro urbano mayor, como la Ciudad lineal de Arturo Soria y Mata respecto a Madrid (I882), o localidades autónomas separadas del núcleo urbano, como el proyecto de la Ciudad Industrial de Tony Garnier (1904). En lo relativo a proyectos de arquitectos

Io. Pani, op. cit., p. 47.

I I. Ortega y Gasset, op. cit., p. Ioo.

I2. Sobre el grado de urbanización de México durante esta época se puede decir que en el Distrito Federal la población aumentó, de 1950 a 1960, de 3.7 a 5.4 millones de habitantes. Esto se debió, en parte, a la acelerada industrialización que experimentó el país a lo largo de esa década, cuya actividad se concentró, con mayor fuerza, en la capital. La ciudad de México se transformó en el principal centro industrial y absorbió, a finales de la década de los cincuenta, " 47 por ciento de la inversión pública federal destinada a la industria, 60 por ciento del renglón de comunicaciones y transportes, y 5 I. 5 por ciento de la inversión total”. Gustavo Garza y Salvador Rivera, "Desarrollo económico y distribución de la población urbana en México, 1960-1990", Revista Mexicana de Sociología. Censos y Población en México, vol. 55, núm. I, enero-marzo de 1990, pp. I77-2I 2.

I3. Pani cita en primer término a Ortega y Gasset: "Yo soy tanto como la suma de mi yo y de mi circunstancia. La disyuntiva de salvarme o perderme es la disyuntiva de salvarme o perderme con ella". Y, después, concluye de manera tajante: "Pues bien, la circunstancia de un hombre es su ciudad. En ella y en su destino está la victoria o derrota del hombre". Pani, op. cit., p. 3. 
modernos, Pani juzga fragmentarias y rígidas las propuestas de Richard Neutra, Walter Gropius y Ernst May, entre otras. ${ }^{14}$ Luego de revisarlas, Pani concluye que la solución de los problemas de la urbe moderna consiste en crear "la ciudad fuera de la ciudad": tal era la consigna. ${ }^{15}$ Con una empresa de ese tipo, según él, se podía enfrentar la problemática del momento: la creciente explosión demográfica de la capital, la creciente demanda de servicios que ella implicaba, la reducción de "espacio vital", el aumento del tráfico, entre otros. Más aún, al planificarse una urbe desde su arranque, se podía llegar a prever su desarrollo físico y demográfico, económico y social, y evitar problemas futuros propios de la gran ciudad. Cabe mencionar que Pani, al efectuar su revisión histórica de proyectos urbanos, destacó la obra de Jean-Gastón Bardet, por impregnar al urbanismo, a través de su topografía humana, de "un sentido social cristiano" ${ }^{16}$ Es importante apuntar que esta referencia aislada a Bardet sitúa a Pani cerca del proyecto de la arquitectura emocional. ${ }^{17}$ Quizá al saber la línea donde se definiría la puerta de entrada a la nueva ciudad, Pani decidió subrayar que sería distinta: un espacio donde se propiciaría la convivencia como punto de partida de una sociedad diferente. Sería una ciudad moderna, aunque revestida con "muros humanos". No está de más mencionar que la imagen de la publicación "México. Un problema, una solución”, que lleva el mismo título, presenta una calle de la ciudad donde la gente camina junto a dos construcciones que remiten, de manera instantánea, al estilo particular de las de Barragán y Goeritz.

I4. Quizá Pani se refiera a los proyectos, respectivamente, de Channel Heights en San Pedro California (I94I-I943), a la colonia Törten en Dessau (I926-I928) y a la Siedlung del Römerstadt en Francfort (1927-1929).

15. Pani realiza incluso un malabar conceptual para justificar históricamente su proyecto al argumentar que a principios de siglo la ciudad de México contaba ya con un sistema de puntos satélite como las zonas de San Ángel y Coyoacán, así como Tacubaya. Pani, op. cit., pp. I9-25.

I6. Ibidem, p. 25.

17. El trabajo de Gastón Bardet no era desconocido, en ese entonces, en México. Pani publicó en 1949 uno de sus textos, "La arquitectura del amor", en la revista Arquitectura-México (núm. 29, octubre de 1949, pp. 204-208), con motivo de su visita a México, donde impartió un ciclo de conferencias en la Escuela Nacional de Arquitectura. No se ha escrito nada sobre la obra de Bardet en relación con el proyecto de arquitectura emocional. Bardet afirmaba que se acercaba una nueva época donde "todo, absolutamente todo será religioso" (p. 205, cursivas del original). En estas condiciones, surgiría una nueva arquitectura que destruiría las diferencias entre tipos de construcciones: "religiosa, civil o militar". Bardet sostenía que la arquitectura tenía que verse como un servicio: un "arte utilitario dirigido al perfeccionamiento del hombre" (p. 205). Esto coincide con la forma en que Goeritz definía su práctica. 


\section{Ciudad Satélite: la construcción incompleta de un proyecto moderno}

Parte del orden urbano que Pani imprimió a Ciudad Satélite refleja algunas de sus preocupaciones básicas sobre el hombre en la urbe. Una de las soluciones más emblemáticas del conjunto cuenta con un criterio social que propicia la convivencia. Ésta es la supermanzana que "concentra en unidades de tipo autónomo, autosuficientes, espacios de habitación y otros tipos de agrupamiento: industrias, escuelas, comercios" ${ }^{18}$ En algunas supermanzanas se construyeron edificios multifamiliares para fomentar, en un espacio común, la asociación de familias de distintas capacidades económicas. En general, la supermanzana pretendía "conservar, reforzar y fomentar el espíritu de asociación". Según Pani, la comunidad que ocuparía Ciudad Satélite sería propia del futuro, y en ella reinaría el "propósito común de resolver problemas sin personalismos, sin particularismos y sin segregaciones", todo con base en la convivencia y el diálogo. ${ }^{19}$ Para Pani, este espíritu de convivencia formaba parte del carácter nacional y del espacio social tradicional de México que a su parecer, un tanto idealista, se conservan aún en los pueblos de provincia. La maniobra persigue una restauración, un regreso a una comunidad orgánica con fuertes lazos sociales que, en apariencia, la modernidad de la gran ciudad desarticuló. La convivencia se garantizaba al hacer de la supermanzana una unidad "centrípeta". ${ }^{20}$ $\mathrm{Al}$ contar con una zona comercial que ofrecería una amplia gama de servicios, el habitante de la supermanzana podría realizar "dentro de ella la mayoría de sus funciones diarias". ${ }^{21}$ Con esto, además, se garantizaría el "espacio vital" del peatón al separarlo, en lo posible, del mundo del automóvil y ofrecerle áreas comunes reservadas para la convivencia. ${ }^{22}$

I8. Pani, op. cit., p. 42.

19. Ibidem, p. 47. Para Pani, el sentido de convivencia y de diálogo en la nueva ciudad no sólo daría como fruto una sociedad, de acuerdo con Ortega y Gasset, sino que, más aún, prefiguraría una especie de paraíso idílico habermasiano regido, sobre todo, por una razón comunicativa.

20. Este modelo es antagónico a la manzana "centrífuga" de la ciudad tradicional que expulsa diariamente a sus habitantes para que realicen sus actividades cotidianas fuera de ella.

2I. Pani, op. cit., p. 44.

22. Es necesario apuntar las similitudes de la supermanzana con las superquadras de Brasilia, la nueva capital de Brasil proyectada por Lucio Costa con arquitectura de Oscar Niemeyer que, coincidentemente, también comenzó a construirse en 1957. La superquadra era asimismo una unidad centrípeta que ofrecía distintos servicios — entre ellos escuelas, comercios y áreas de recreación-y segregaba el tráfico vehicular hacia el exterior de la cuadra. Más aún, la superquadra también perseguía el propósito de crear "nuevos patrones de interacción social”. Véase Valerie 
2. Satélite como una ciudad con "muros humanos". Imagen tomada de Pani, "México. Un problema, una solución", op. cit. (supra n. 8), p. I4. Fondo Mathias Goeritz. Archivo Cenidiap/InBa.

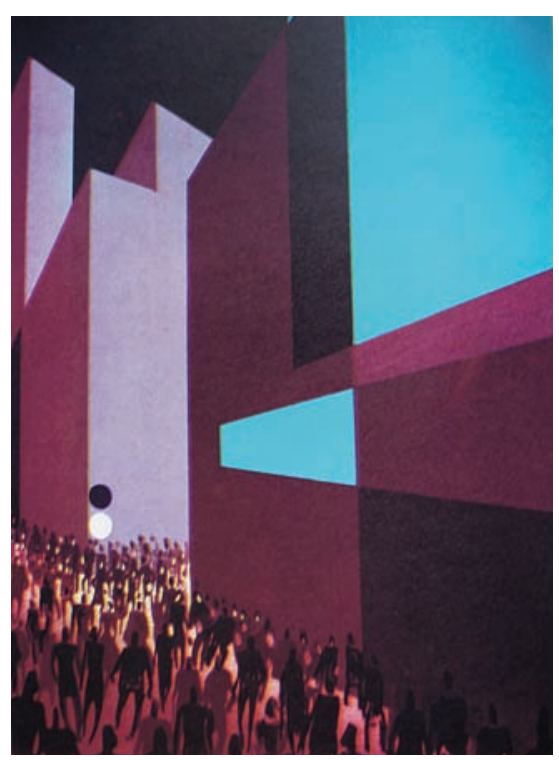

La dinámica del automóvil se organizó en Ciudad Satélite de acuerdo con el Sistema Herrey, utilizado por Pani por vez primera cuatro años atrás en el proyecto de Ciudad Universitaria. Este modelo se caracteriza por calles de un solo sentido, sin interrupciones por cruceros, que, al aspirar a una vialidad continua, rechazan el trazo tradicional en línea recta. La idea es que se entrara a ese circuito vial, y así a Ciudad Satélite, a través de unos pasos a desnivel ubicados en dos puntos del Eje Central marcados por un par de plazas monumentales. Las Torres corresponden al proyecto de la Plaza Sur y la Plaza Norte no se construyó jamás. Las previsiones de estos pasos a desnivel en el texto de la conferencia "México. Un problema, una solución", de Mario Pani, poseen un aspecto aerodinámico que recuerda la tendencia constructiva streamline. Esto es más evidente en la estructura de color rojo, lo que bien podría ser un puente peatonal, con el énfasis de sus curvas y su largo trazo horizontal. Esta estructura, tal como aparece en la ilustración, reviste un carácter abstracto que la acerca a un objeto escultórico. El estilo streamline alcanzó su máximo apogeo a finales de la década de los años treinta, tal como se puso de manifies-

Fraser, Building the New World. Studies in the Modern Architecture of Latin America, 1930-1960, Londres, Verso, 2000, pp. 235-237. 
to en la Exposición Mundial de Nueva York (1938-1939), y desde siempre se asoció con valores próximos a lo moderno, como el dinamismo y la velocidad, atributos de los que también se buscaba dotar a la nueva Ciudad Satélite. ${ }^{23}$

Queda claro que, desde su ubicación hasta su planificación y organización, Ciudad Satélite se centra en el uso del automóvil y depende de él. Esto también lo vuelve un proyecto distintivamente moderno. Aunque Pani no opta por la vialidad como una línea recta con cruces, constante en la mayoría de las propuestas de urbanismo moderno, sí comparte la idea moderna de que la prosperidad de una ciudad depende de la velocidad que se alcance. ${ }^{24}$ Según Pani, su época era la del automóvil, y Ciudad Satélite, con un sistema vial de circulación continua, respondía a ello. ${ }^{25}$ Así se evitaría el tráfico que se traduce en pérdida de tiempo y dinero. Para Pani el Sistema Herrey solucionaría el problema de la creciente motorización del México de los años cincuenta. Muchos arquitectos modernos — principalmente Le Corbusier - considerarían pintoresco el trazo del Sistema Herrey, caracterizado por líneas curvas. Sin embargo, de tal característica se derivan varias virtudes de dicho sistema: una mayor seguridad para el automovilista, una negociación y adaptación más adecuada a las condiciones del terreno y el auto como "instrumento para generar la percepción dinámica del paisajismo generoso" ${ }^{26}$

La creciente motorización de México ocurrida a partir de inicios de la segunda mitad del siglo xx no es un caso aislado, sino un fenómeno más amplio registrado en la mayor parte del mundo occidental. Esto es reflejo, en parte, de un proceso de modernización caracterizado por seguir patrones del modelo estadounidense. ${ }^{27}$ El proyecto de Ciudad Satélite parece responder a esto en más de un sentido. Originalmente, Pani planteó la posibilidad de hacer del centro comercial uno de los espacios comunales de mayor importancia. En su proyecto, esta tipología, desarrollada en Estados Unidos en la

23. Véase Erik Mattie, World's Fairs, Nueva York, Princeton Architectural Press, I988, pp. 195-199.

24. Le Corbusier, The City of To-Morrow and its Planning, Nueva York, Dover, 1987, pp. 190I9I (publicado en 1924 como Urbanisme).

25. Pani, op. cit., p. 49.

26. Peter Krieger, "Hermann Zweigenthal-Hermann Herrey. Memoria y actualidad de un arquitecto austriaco-alemán exiliado", Anales del Instituto de Investigaciones Estéticas, vol. XXVI, núm. 85 , otońo de 2004 , p. 7 .

27. Respecto al caso específico de Francia, véase Kristin Ross, Fast Cars, Clean Bodies. Decolonization and the Reordering of French Culture, Cambridge, MIT Press, I999. 
década de los cincuenta, sería el nuevo espacio civil para Ciudad Satélite, sitio enmarcado por actividades de consumo. Este espacio se había concebido para satisfacer la demanda de servicios que no tuviera cabida en las supermanzanas. En el conjunto del complejo comercial habría establecimientos diversos, dos grandes tiendas departamentales, un par de supermercados, un centro de negocios con área de oficinas, instalaciones de consultorios médicos, cines, teatros, gasolineras e inclusive una terminal de autobuses..$^{28}$ En este espacio, una serie de actividades cotidianas se organizarían con base en el consumo. Este centro comercial nunca se construyó. Sin embargo, es importante destacar que, en la fecha de su concepción, lo que Pani proponía se adelantaba a los proyectos e ideas que James Rouse desarrollaría en los años sesenta. A Rouse, uno de los mayores constructores de proyectos de índole comercial en Estados Unidos, se le atribuye la creación del modelo de centro comercial anticipado por Pani, donde se ofrece un sinnúmero de servicios para satisfacer las complejas demandas de la comunidad y se mantiene una base estable de consumidores. ${ }^{29}$

Durante mucho tiempo, Ciudad Satélite contó sólo con Centro Satélite, que era, básicamente, un supermercado. No será sino hasta principios de los años setenta, una vez que el proyecto original de Ciudad Satélite ya se había desechado, cuando se construyó un centro comercial en plena forma. Plaza Satélite (1968-197I) es una obra del arquitecto Juan Sordo Madaleno de menores ambiciones que el proyecto de Pani, la cual, en términos generales, sigue de manera convencional la topografía del centro comercial suburbano de Estados Unidos planteada por vez primera por Víctor Gruen en los años cincuenta. Hoy en día, el proyecto de Pani relativo al centro comercial podría parecer visionario por anticiparse a proyectos de arquitectura contemporánea que giran alrededor del fenómeno de la congestión. ${ }^{30}$

28. Pani, op. cit., pp. 47-49.

29. Un caso de esto sería el proyecto de Rouse para expandir el "Cherry Hill Mall” (I972). Aunque Welton Beckett había propuesto en I954 un centro comercial con hospital, éste nunca se construyó. Véase Stephanie Dyer, "Designing 'Community' in the Cherry Hill Mall. The Social Production of a Consumer Space”, Perspectives in Vernacular Architecture, vol. 9, Constructing Image, Identity and Place, 2003, p. 267. Aunque el desarrollo del modelo del centro comercial suburbano es producto del trabajo de Víctor Gruen, Rouse es el primero en visualizar un espacio que, además de contar con comercios, ofrecería hospitales y espacios para oficinas, entre otros servicios.

30. Un ejemplo de esto lo sería el trabajo del arquitecto Rem Koolhaas. Véase su Delirious New York. A Retroactive Manifesto of Manhattan, Nueva York, The Monacelli Press, I994. 


\section{Un símbolo plástico-publicitario para Ciudad Satélite}

Entre lo poco que se materializó, de todo lo que anticipaba el proyecto original de Ciudad Satélite, se encuentran las Torres. En su propuesta inicial, Pani concibió dos plazas monumentales, situadas sobre el Eje Central, que marcarían los límites de Ciudad Satélite al sur y al norte, y serían así a la vez puertas y símbolos del conjunto. Al hacer referencia a estas plazas de grandes proporciones debe recordarse el interés que por la monumentalidad y la figura del monumento manifestaba el arquitecto Pani, quien ya había ganado un concurso para diseñar el Monumento al Himno Nacional y había participado en otro convocado por Excélsior, donde alcanzó un segundo lugar. Cabe mencionar que en estas propuestas se aprecia un interés por la verticalidad, lo cual se revela de manera más clara en el primer proyecto que, dicho sea de paso, no se construyó. El uso de estructuras verticales que centran la atención en el espacio es apreciable en algunas obras de Pani como, por ejemplo, la Escuela Nacional de Maestros (1945). También conviene considerar que Pani se inclinaba por la "integración plástica", es decir, por nuevas formas de relacionar la arquitectura con la escultura y la pintura. ${ }^{3 \mathrm{I}}$ En esta línea, es preciso aclarar que en los proyectos de Pani, la mayor parte de las veces, las intervenciones realizadas por los artistas no pueden considerarse, en sentido estricto, figurativas ni cercanas al realismo que caracterizó a la Escuela Mexicana. Un caso que ilustra esto sería el mural realizado por Roberto Engelking en el auditorio al aire libre del Conservatorio Nacional de Música (1946). Esto no quiere decir que la producción careciera de un contenido nacionalista, tal como se evidencia en varias obras que realizó para el Estado. El mural con el tema del mestizaje realizado por José Clemente Orozco en la Escuela Nacional de Maestros y los paneles de inspiración prehispánica de Carlos Mérida en el multifamiliar Presidente Juárez (I95I) son dos ejemplos de "integración plástica” en los proyectos de Pani.

Quizá gracias a esta primera especialización en monumentos conmemorativos, Pani llamó a Barragán, quien a su vez invitó a Goeritz, para diseñar un espacio distintivo, la Plaza Monumental Sur de la nueva ciudad. Así, el autor del proyecto canceló la posibilidad de introducir referentes monumentales tradicionales, de sentido nacionalista, en la que sería su urbe del futuro. Como se sabe, el trabajo de Barragán y Goeritz era apreciado en esta época por el lenguaje 
3. Ilustración de los pasos a desnivel en Ciudad Satélite. Imagen tomada de Pani, "México. Un problema, una solución”, op. cit. (supra n. 8), p. 50. Fondo Mathias Goeritz. Archivo Cenidiap/InBa.

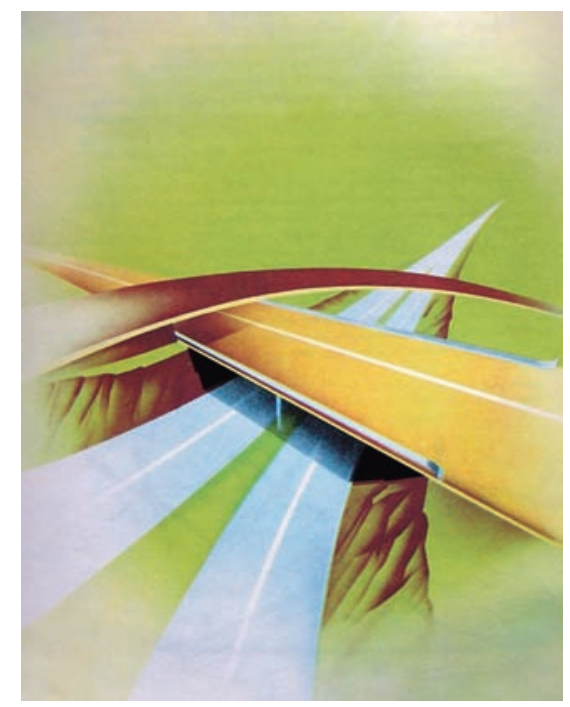

figurativo, la puesta al día de la tradición, las formas que tendían a la abstracción y la distancia respecto a contenidos nacionalistas. La invitación referida cobra mucho más sentido si se considera la crítica positiva y el éxito comercial que, en el ámbito nacional e internacional, recibieron y alcanzaron, respectivamente, los Jardines del Pedregal, donde siempre se subrayó el diseño de la plaza de acceso en la avenida de las Fuentes, con El animal del Pedregal de Goeritz, y el de los jardines de uso comunal. No debe olvidarse que en su proyecto original la Puerta Sur de Ciudad Satélite funcionaría como una plaza pública. Su planeada monumentalidad, tal como dijo Pani, tenía el objetivo de que el conductor redujera la velocidad al observarla, lo cual facilitaría el acceso al circuito interior de Ciudad Satélite por el paso a desnivel. ${ }^{32}$ Además de realizar esta función, Pani pidió que en su proyecto la escala se entendiera de una nueva forma: alejándose de la escultura conmemorativa, buscaba ser símbolo de "ese propósito incoercible del hombre que trasciende en las grandes cosas que parecen inútiles, pero que representan la presencia del espíritu y de la dignidad en las obras humanas". ${ }^{33}$ Esta nueva definición de monumentalidad, hasta donde se puede apreciar, no contradice los objetivos del proyecto de la arquitectura emocional.

32. Pani, op. cit., p. 5 I.

33. Ibidem, p. 53 . 
El primer modelo con que se presentan las Torres contaba con las cinco estructuras verticales de planta triangular distribuidas en una explanada articulada en tres desniveles conectados por escaleras. Estos desniveles constituirían plataformas que estarían alfombradas con pasto; además, en una de ellas se planeaba construir un espejo de agua de grandes dimensiones. ${ }^{34}$ Goeritz comentó que la intención inicial era contar con siete en vez de cinco torres. Sin embargo, por cuestiones económicas sólo se construyeron cinco. Este factor también influyó en la altura de los cuerpos, pues en un inicio la más alta llegaría a 200 metros. ${ }^{35} \mathrm{Al}$ final, sólo alcanzó 57, en lo que podría ser una referencia al año de su construcción. Se puede especular sobre las razones del cambio del diseño original al alterarse el conjunto de la planta y eliminarse el espejo de agua. Quizá se debió a la previsión de que en el futuro surgiría una serie de inconvenientes en una plaza pública situada en medio de una arteria vial que, con el tiempo, sería altamente concurrida y de alta velocidad. Es en este momento cuando se deja de definir este espacio como plaza y se empieza a referir como glorieta.

De acuerdo con Goeritz, la construcción de las Torres comenzó en mayo de 1957 y concluyó en noviembre de ese año. El ingeniero a cargo de la construcción fue Bernardo Quintana. Las Torres fueron coladas por segmentos que crearon la textura, a manera de estrías, lo cual las caracteriza. En agosto se reportó un problema estructural en una de ellas (la denominada E). La construcción se detuvo y se corrigió una torsión que, de otra forma, hubiera parecido a simple vista un desplome de la estructura. Reparar esta falla era imperativo para el Comité Directivo de Ciudad Satélite, pues se temían "los efectos psicológicos que pudiera representar para la venta". ${ }^{36}$ Esta cuestión adquiere mucho más sentido si se recuerda que semanas antes, el 28 de julio, la ciudad de México fue sacudida por un sismo de 7.5 grados que causó el derrumbe de edificios y casas, así como la caída del Ángel de la Columna de

34. Mathias Goeritz llegó a comentar que el cambio en la plataforma de las Torres fue una decisión suya que buscaba acentuar el efecto de las esculturas al eliminar cualquier elemento que embelleciera el conjunto. Véase Carl Nielsen, "Gateway to the City", Dimension. Student Publication, Ann Arbor, University of Michigan-College of Architecture and Design, núm. I, I958, pp. I5-I6.

35. Mathias Goeritz, "Highway Sculpture: the Towers of Satelite City", Leonardo, vol. 3, julio de I970, p. 321 .

36. Luis García Barbachano, "Informe de la sesión XX del Comité Directivo de Ciudad Satélite del Banco Internacional Inmobiliario, S.A.", México, 23 de agosto de 1957. 
la Independencia, en el Distrito Federal. ${ }^{37}$ Contra el panorama de una ciudad colapsada, el continuo ascenso de las Torres pudo ser un signo de estabilidad, seguridad y perdurabilidad de la nueva Ciudad Satélite.

En noviembre, las Torres ya se habían construido tal como las conocemos hoy, pero aún no se pintaban. El conjunto consta de cinco estructuras verticales monumentales de planta triangular, cuyo vértice más agudo apunta hacia el Distrito Federal. Sus alturas varían, pues oscilan de los 57 metros de la más alta a los 37 de la más corta. La explanada la constituye una plancha de concreto de forma oval donde las Torres se encuentran distribuidas de manera irregular y que, en un principio, tuvo carácter de glorieta. A sus lados, la carretera se mueve hacia el norte y el sur. Una vez terminadas las torres, se tuvo que esperar tres meses con el fin de que el concreto secara completamente para pintarlas. Al principio se eligió para hacerlo una gama de rojos. Sin embargo, por una cuestión económica relacionada con los costos de la iluminación, se decidió pintar tres torres de blanco, una de amarillo y otra de rojo. ${ }^{38}$ Éstos fueron los colores con que las Torres se presentaron por vez primera durante los meses iniciales de 1958. Goeritz comenta que por una decisión arbitraria, a finales de 1958, una de las torres blancas se pintó de azul. Esto constituía un error para Goeritz, ya que la estructura no contrastaría con el cielo en el horizonte. ${ }^{39}$ Goeritz también procuró materializar la idea original de instalar una serie de flautas y silbatos encima de las Torres para que el viento produjera ciertos sonidos. Pero los promotores la desecharon. ${ }^{40}$ Los silbatos y flautas, sin embargo, revelan el interés de Goeritz, durante esta época, por un tipo de escultura relacionada de forma abierta y dinámica con el viento.

Si se considera la relación de las Torres con el ente articulador de Ciudad Satélite, el automóvil, se puede apreciar una preocupación claramente moderna en la proyección del conjunto. La segunda torre más alta, pintada de color rojo, ubicada al frente de las otras cuatro, produce al acercarse a ella en el automóvil desde el Distrito Federal un efecto óptico que imprime cierto

37. Linda Manzanilla, "Relación de los sismos ocurridos en la ciudad de México y sus efectos", Revista Mexicana de Sociología. Sismo, Desastre y Sociedad en la Ciudad de México, vol. 48, núm. 2, abril de 1986, p. 280.

38. Reporte de la empresa Luminotecnia, firmado por su gerente Alfonso Bustamante, dirigido al Banco Internacional Inmobiliario y al arquitecto Mario Pani y fechado el I9 de agosto de 1957.

39. Goeritz, op. cit., p. $32 \mathrm{I}$.

40. Idem. 


\section{42}

movimiento al conjunto. Más aún, críticos de la época describen las múltiples impresiones del mismo tipo que se pueden producir al dirigir a esta obra monumental una mirada en movimiento. Nesbit, por ejemplo, comentó que los efectos son los de un constante cambio de sus alturas y ángulos que llevan a verlas, en un momento, como estructuras delgadas, después como placas y, finalmente — si se ven desde el norte_- como rectángulos..$^{4 \mathrm{I}}$ Este potencial del automóvil y del espectador móvil confiere a las Torres de Satélite una característica que hace de ellas una obra provista de un distintivo carácter moderno, que se resume en la consideración del efecto de los nuevos medios de transporte en la percepción visual a través de una solución que se adapta a esta nueva forma de observar. ${ }^{42}$ No es que investigaciones de este tipo no hubieran tenido lugar en México. Un antecedente de él sería el mural de David Alfaro Siqueiros El pueblo a la Universidad, la Universidad al pueblo, ubicado en el costado sur de la Torre de Rectoría de Ciudad Universitaria. Sin embargo, los volúmenes y la proporción de las torres convierten a este grupo escultórico y a la experiencia de mirarlas desde una perspectiva móvil en algo único y sin precedentes en su tiempo.

Además de lo anterior, las Torres son una forma arquitectónica moderna íntimamente ligada con la publicidad. Barragán, por ejemplo, las llegó a denominar un "símbolo plástico publicitario". ${ }^{43}$ Este uso de la arquitectura y el arte moderno como publicidad no era, en los años cincuenta, nada nuevo, como el mismo Barragán y Goeritz sabían bien. ${ }^{44}$ Aunque sus autores declararon que la única función de las Torres era de carácter emocional, no se puede dejar de mencionar que este conjunto escultórico sí actuaba como puro valor de cambio, a la manera de la publicidad. Una vez construidas, se podría decir que

4I. G. Nesbit, "The Towers of Satelite City", Arts and Architecture, Los Ángeles, vol. 75, núm. 5, mayo de 1958, p. 22.

42. Beatriz Colomina, Privacy and Publicity. Modern Architecture as Mass Media, Cambridge, MIT Press, I996, p. 6I. Este tema ha sido analizado en profundidad por Krieger, "Potencial simbólico...", op. cit., pp. 197-198 y 206-207.

43. Luis Barragán, "La propiedad artística de las Torres de Satélite", Plural, Revista Cultural de Excélsior, núm. 48, septiembre de 1975, Sección Correspondencia, p. 84.

44. Véase un debate sobre la relación entre la arquitectura y la publicidad en la obra de Barragán en Keith Eggener, "La 'arquitectura fotográfica' de Barragán: imagen, publicidad y memoria”, en Federica Zanco (ed.), Luis Barragán. La revolución callada, Milán, Skira, 200I. El nexo de la publicidad con las Torres de Satélite ha sido abordado por Krieger, "Potencial simbólico...", op. cit., pp. 213-216; también es un tema que he tratado en mi artículo "Las Torres de Satélite...", op. cit. 
4. Diagramas que ilustran el Sistema Herrey. Imagen tomada de Pani, "México. Un problema, una solución", op. cit. (supra n. 8), p. 52. Fondo Mathias Goeritz. Archivo Cenidiap/InBA.

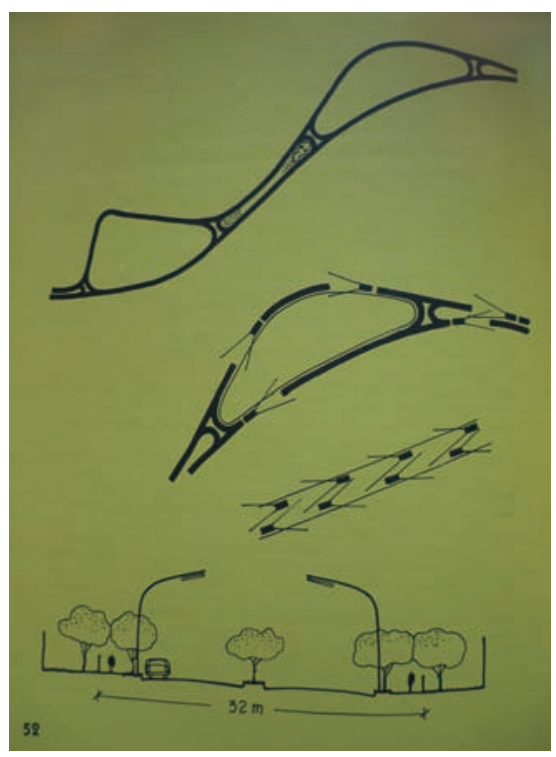

su papel como anuncio fue contundente con relación al éxito comercial del fraccionamiento. Las expectativas de sus inversionistas y promotores fueron rebasadas rápidamente. Al inicio de I958, había ocho casas en Ciudad Satélite y se esperaba que en diciembre — conforme a un cálculo más que optimista- hubiera ya varias centenas de construcciones. ${ }^{45}$ Es quizá esta fortuna comercial lo que llevó a Pedro Friedeberg a concluir en I96I su "Relato histórico de la Torre" con un tono un tanto irónico y con el llamado "a los arquitectos y al pueblo de México en general, a seguir la alta tradición del pasado. Hay que construir torres cada vez más altas para el deleite del espíritu, del alma y de los negocios".${ }^{46}$ Como se puede apreciar, los objetivos de la arquitectura emocional no riñeron con el mundo de la compra y venta de bienes raíces. La crítica que se podría dirigir al "negocio de la arquitectura emocional" continuó y se intensificó en los comentarios de Mauricio Gómez Mayorga, quien atribuyó a las Torres una práctica de "arquitectos dibujantes y publicistas" a la que llamó

45. "Autonomía urbana. Espárragos a la entrada", Visión, Nueva York, 3I de enero de 1958 , sección "Urbanización", p. 44-45.

46. Pedro Friedeberg, "Relato histórico de la Torre", Arquitectura-México, año XXIII, t. XVII, núm. 73, marzo de I96I, p. 55. 


\section{44}

DANIEL GARZA USABIAGA

"cartelismo en tres dimensiones". ${ }^{47}$ Para él, la obra era la peor muestra de todo el proyecto colaborativo de la arquitectura emocional.

\section{Sobre la Plaza Monumental Norte}

Aunque prácticamente desconocido, sí llegó a haber un anteproyecto de la Plaza Monumental Norte. Todo parece indicar que esta construcción fue obra exclusiva de Goeritz, según se puede demostrar a partir de cierta evidencia. En la reimpresión de la conferencia "México. Un problema, una solución" de Pani, que se difundió en el sobretiro del número 60 de la revista ArquitecturaMéxico, aparecen, una vez que se discuten las dos plazas monumentales en el texto, la fotografía de la primera maqueta de las Torres y un dibujo que, presumiblemente, representa la segunda plaza. Esta obra consta de un muro de proporciones colosales, a la manera de un ciclorama, sostenido a través de pilotes que descansan en una fuente circular de gran diámetro. Sobre esta fuente, en su centro, aparece un volumen esférico que parece tener en su superficie ciertos orificios o perforaciones que la muestran hueca. En una publicación de 1958 apareció una descripción más detallada de este proyecto que, como casi todo en la propuesta inicial de Pani, nunca se concretó. En una entrevista con Goeritz, se señala que el espacio de la Plaza Norte estaría definido por un muro de 35 a 40 metros de altura y 250 de largo, suspendido del piso por unos pilotes que resultarían invisibles detrás de los chorros de agua de la fuente. $4^{8}$ La pared sería curva, con el fin de eliminar completamente la visión del paisaje. El muro lo ocultaría detrás de los límites de Ciudad Satélite y, de manera inversa, antes de ingresar a ella viniendo por la carretera a Querétaro, hurtaría a la mirada toda la ciudad. Como en otras obras de Goeritz, este muro parece contemplar en su proyección un momento donde algo que ha permanecido oculto aparece repentinamente y de manera sorpresiva, causando cierto efecto en el observador. El muro puede verse, pues, como colofón de la ciudad con torres: la urbe amurallada. Con relación al mencionado elemento esférico que el dibujo sitúa al centro de la fuente, Pani comentó que, en un proyecto de

47. Mauricio Gómez Mayorga, "La arquitectura contemporánea en México. Notas polémicas", Artes de México, núm. 36, año IX, octubre de I96I, p. I I. Por carecer de función, Gómez Mayorga consideró a las Torres estructuras "ociosas" (p. I2).

48. Nesbit, op. cit., p. 22. 
construcción de una ciudad satélite en Caracas, Venezuela, propuso, junto a Mathias Goeritz, "unos elementos o esferas movidas por el viento, en ciertos edificios, como un ensayo interesante de integración plástica”. ${ }^{49}$ No cabe duda de que el elemento esférico en el dibujo publicado en el sobretiro de la revista Arquitectura-México puede ser una representación preliminar de ese tipo de esculturas. La idea de un muro de dimensiones colosales parece haber encontrado su concreción final en la pieza que Goeritz realizó para la Unidad Adolfo López Mateos en el Estado de México (1964), proyecto habitacional que en su tiempo se consideró "la primera ciudad de interés social". A diferencia del proyecto de Ciudad Satélite, donde la plaza monumental se encontraría en un espacio caracterizado por el paisaje abierto, en la unidad habitacional el muro se encuentra en el centro, delimitando la Plaza Cívica. Debido a su ubicación dentro del conjunto, la experiencia con esta estructura, por su escala y proporción, resulta del todo distinta de la proyectada originalmente en Ciudad Satélite. El muro, en la Unidad Adolfo López Mateos, se pintó de color amarillo y soportaba, en su parte inferior, un relieve con el nombre del conjunto habitacional. En vez de contar con una fuente y un objeto esférico, se relaciona con una obra de Carlota Yazbeck donde un grupo de tres figuras antropomorfas remite a la imagen de la Sagrada Familia.

\section{Las Torres como un elemento de la arquitectura emocional}

¿De dónde proviene y cómo se puede entender el uso del motivo de la torre? En términos de construcción, y como un rasgo definitorio del conjunto escultórico, se puede decir que en 1957 Goeritz y Barragán habían iniciado ya, en sus obras particulares, una investigación sobre los efectos de la verticalidad en sus proyectos. ${ }^{50}$ De la misma forma, contaban con antecedentes autobio-

49. Véase la cita de Pani en Mario Pani..., op. cit., p. 53.

50. Goeritz remonta su interés por el tema de la torre en su carrera a la construcción de su Museo Experimental El Eco, con la torre amarilla ubicada en el patio. Posteriormente, se refiere a la escultura Aqui y allá (1954-1955), donde una serie de elementos verticales se contrastan con volúmenes mucho más pequeños. Se podrían dar más ejemplos pues, como se sabe, el tema de la torre es una constante a lo largo de la producción artística de Goeritz. Barragán, por su parte, aunque sin hacer referencia directa a la torre, había emprendido ya en 1957 una investigación sobre la verticalidad. Esto comienza en su casa de Tacubaya (I947-I948), específicamente en la azotea-terraza, y se manifiesta mediante volúmenes verticales prominentes que confieren a 
gráficos, un recurso muy usado en el análisis de sus obras, que manifestaban su fascinación por el motivo de la torre. Barragán se refiere a las torres de Bolonia y San Gimignano, en Italia, país que visitó en 1952. Goeritz compartía esta particular predilección, así como una atracción temprana por el paisaje de los rascacielos de Manhattan. Respecto a la biografía de los autores, es probable que Goeritz, particularmente, haya tenido conocimiento de algunas propuestas de corte expresionista de arquitectos alemanes antes de su exilio. Hay toda una serie de proyectos asociados con esta vanguardia histórica, donde aparecen construcciones semejantes a las estructuras de las Torres de Satélite. El proyecto, no realizado, de un rascacielos en Friedrichstrasse, de Mies van der Rohe (I92I), y la ilustración de Peter Behrens para la portada del número 6 de la revista Das Plakat (junio de 1920) son un par de ejemplos al respecto. La semejanza radica en la verticalidad de las estructuras y en su planta triangular, debido a la cual, al mirarlas de frente, parecen rematar en una punta en la parte superior. Dentro del contexto expresionista, estas construcciones remitían a la figura y a todo el simbolismo del cristal —relacionado con valores de pureza, orden e individualidad - e intentaban recuperar el complejo significado medieval del intento de unir en ellas el dominio de lo celestial y lo terrenal. ${ }^{51}$ El contexto expresionista también vincula el elemento de la torre con la experiencia de lo sublime. En general, se puede decir que el expresionismo consideraba, tal como lo postuló Edmund Burke en su Indagación filosófica sobre el origen de nuestras ideas acerca de lo sublime y de lo bello (1756), ciertos fenómenos y objetos que, por sus características específicas — relacionadas con la grandeza, la infinidad, la magnitud y la vastedad-, eran capaces de provocar en el espectador, de manera instantánea, emociones de asombro, admiración y sorpresa. Burke mismo utilizó el motivo de la torre para ilustrar tal idea en su texto; para él, ciertos elementos arquitectónicos son suficientemente evocativos de varias emociones que satisfacen las demandas de lo "sublime". 52

este espacio un carácter plástico. Otro proyecto de Barragán que expresa el mismo interés es la Plaza del Cigarro (1952), donde se usa un elemento vertical para marcar una zona, la comercial, dentro de El Pedregal. Como puede verse, el afán de verticalidad, que en las Torres se lleva a una proporción colosal, es un rasgo común a Goeritz y a Barragán

5I. El contexto biográfico temprano de Mathias Goeritz y su relación con las Torres de Satélite ha sido discutido por Krieger, "Potencial simbólico...", op. cit., pp. I85-198.

52. Véase Katarzyna Murawska, "An Image of Mysterious Wisdom Won by Toil: The Tower as a Symbol of Thoughtful Isolation in English Art and Literature from Milton to Yeats", Artibus et Historiae, vol. 3, núm. 5, I982, p. I42. 
5. Desarrollo de Ciudad Satélite con supermanzanas. Imagen tomada de Pani,

"México. Un problema, una solución",

op. cit. (supra n. 8), p. 43. Fondo

Mathias Goeritz. Archivo Cenidiap/InBa.

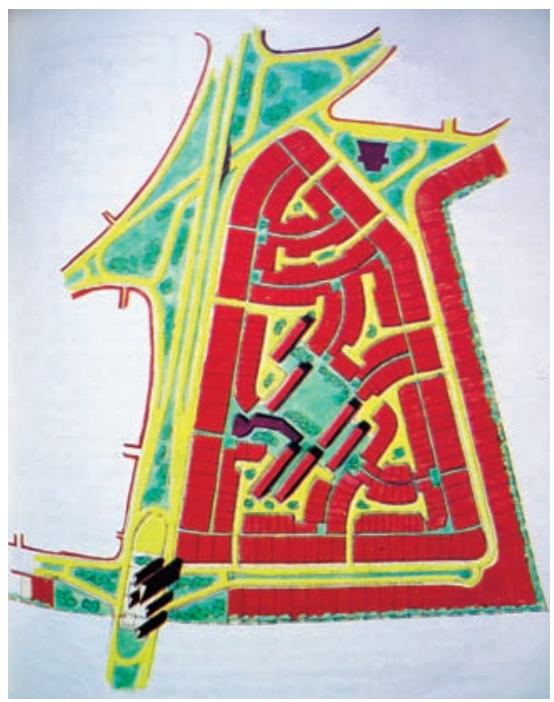

Más allá de la controversia sobre los créditos de la autoría de las Torres, que es de dominio público, no hay duda de que tanto Goeritz como Barragán consideraron esa obra un ejemplo de la arquitectura emocional. Aunque se llegó a especular que una de las torres funcionaría como observatorio mientras otras servirían para colectar agua, la construcción no tiene en realidad ningún uso funcional. ${ }^{53}$ Su único propósito es emocional, como diría Goeritz, o poético, en palabras de Barragán. En la magnitud del conjunto, tal como se situaba en el paisaje, reside la posibilidad de que provoque emoción, ya que es una estructura que mueve, cuando se la descubre, al asombro, seguido de un placer derivado de una contemplación más minuciosa. Es de esta forma, especialmente con relación a su contexto original y la dimensión de su escala, como el conjunto escultórico se liga con la categoría de lo sublime, como un - y sólo un- modo de entender la esfera afectiva. ${ }^{54}$ La superficie de las

53. "To Mark Mexican Satellite City", Progressive Architecture, Nueva York, octubre de I957, p. 94. 54. Aunque éste no es el sitio para ahondar al respecto, es necesario mencionar que, si bien Goeritz siempre sostuvo que el principal objetivo de sus proyectos era provocar una emoción, prácticamente nunca avanzó más allá de esto en su discusión. Así, el entendimiento y la reflexión de la esfera afectiva en los escritos de Goeritz es, en comparación con cierta literatura de la época, un tanto limitada. Pareciera que Goeritz, al declarar la función emocional de su arquitectura, adoptara un concepto derivado de Edmund Burke, especialmente de su A Philosophical Enquiry 
construcciones, caracterizada por la textura y las estrías visibles, presenta un aspecto irregular y suscita un efecto táctil que da a las Torres un carácter único y fuertemente expresivo. Por su irregularidad y su aspecto material podría considerárseles, también un elemento pintoresco del paisaje. El modo de edificarlas, además, las vinculó con una tradición constructiva del país, que confiere a las estructuras cierto aspecto regional.

El uso del referente de las torres, por parte de los autores, dota a la obra de múltiples significados y pone de manifiesto la importancia atribuida a la categoría del símbolo en el comportamiento humano. ${ }^{55}$ Desde siempre - y aquí se puede pensar en el ejemplo de la Torre de Babel—, la torre cifra relevantes significados simbólicos, principalmente como puente o estructura que aproxima el mundo terrenal al sacrum de los cielos. La experiencia con ellas, por lo tanto, es más cercana a la mística que a la razón y a la lógica pura. ${ }^{56}$ Esta lectura del motivo de la torre coincide con el programa de la arquitectura emocional. Una interpretación moderna de este elemento arquitectónico se encuentra también en conexión con tal programa de arquitectura. La torre, principalmente a partir de la literatura del siglo XvIII, se concibe como un espacio de retiro para la meditación solitaria y el aislamiento filosófico. Katarzyna Murawska ha analizado este concepto de la torre, cuyo origen se remonta al siglo XVII, con Il penseroso (I63I) de John Milton, y lo encuentra expresado todavía, a finales del XIx, en obras como La-Bas (I89I) de Joris-Karl Huysmans. Sin embargo, no se puede dejar de mencionar que la torre, como motivo, tiende a ser dual y posee un doble filo, en parte debido a las complejas contradicciones que encierran elementos relacionados, de una u otra manera, con la lógica del romanticismo. Como la misma historia de la Torre de Babel atestigua, esta estructura también es un símbolo que surge de la vanidad y el orgullo. Una vez en ruinas, se vuelve, dentro de la lógica del paisaje

into the Origin of our Ideas of the Sublime and Beautiful (Oxford University Press, 1998), donde la experiencia estética o respuesta emotiva es inmediata: la naturaleza y las obras de arte afectan la sensibilidad directamente, sin mucha intervención del pensamiento racional. Esta perspectiva también se manifestó, como se menciona en el texto, en el grupo de vanguardias históricas alemanas asociado con el "expresionismo". Véase Anthony Quinton, "Burke on the Sublime and Beautiful”, Philosophy, vol. 36, núm. I36, enero de 1961, p. 72.

55. Con base en el cuerpo de su obra, se puede decir que una consideración sobre la importancia del símbolo en el comportamiento humano es una constante en la obra de Goeritz, y no en Barragán.

56. Murawska, op. cit., p. I48. 
pintoresco, una especie de vanitas. En cuanto a la forma en que se la concibe en la literatura moderna, Murawska ha explicado cómo la reflexión filosófica se puede tornar en escapismo, en el aislamiento que dispensa la "Torre de Marfil", como un espacio de confinamiento que niega la realidad existente. ${ }^{57}$ Murawska concluye en su estudio que en el siglo xx William Butler Yeats desarticula y pone en entredicho, de manera contundente, el entendimiento de la torre como un espacio de reclusión filosófica y reflexión.

Durante la primera mitad del siglo xx, la forma de la torre, transformada por la práctica arquitectónica, se asociará con la construcción de rascacielos. Se abre así una historia particular que, en sí, deja de asociarse con este motivo propio del pasado. Algunas vanguardias históricas, sin embargo, siguieron haciendo uso en sus obras del motivo de la torre durante las primeras cuatro décadas del siglo xx. Ejemplos de esto son el expresionismo - que ya se señaló-y el surrealismo que, más que visualizarlas o construirlas, las rescataba en su forma de ruinas urbanas. ${ }^{58}$ Más allá de estos ejemplos, se puede decir que, en los inicios de la segunda mitad del siglo pasado, el motivo de la torre se encontraba prácticamente en desuso y, en más de un sentido, desarticulado. Lo mismo es posible afirmar respecto a la intención de evocar un estado afectivo que se asociara, de manera muy directa, con la categoría de lo sublime, especialmente después de su empleo dentro de distintos procesos de estetización de la política y creación de espectáculos. ${ }^{59}$ En este contexto, ¿qué significa la

57. Ibidem, pp. I53-159.

58. Véase, por ejemplo, André Breton, Mad Love, Lincoln, University of Nebraska Press, 1987, pp. 47-49 (publicado originalmente como L'Amour Fou, 1937).

59. Como Neal Wood ha explicado, Burke veía la categoría de lo sublime como un ingrediente vital de la autoridad política. La representación y la puesta en escena de la magnificencia y el poder de la autoridad en cuestión, comparadas con el uso abierto de la fuerza bruta, no acarrean consecuencias violentas o capaces de debilitar la fábrica social. Para Burke, la admiración era el primer paso rumbo a la obediencia. "Grandeza y esplendor en los discursos, edificios, parafernalia ceremonial y sus actos son todos puntos de partida de lo sublime en la autoridad política." Véase "The Aesthetic Dimension of Burke's Political Thought", The Journal of British Studies, vol. 4, núm. I, noviembre de 1964, pp. 58 y 60. Cabe mencionar que Burke fue capaz de vislumbrar los problemas de abuso de esta autoridad política a través de un sublime "falso". Véase Stephen White, "Burke on Politics, Aesthetics, and the Dangers of Modernity", Political Theory, vol. 2I, núm. 3, agosto de 1993, pp. 507-527. En esta misma línea cabría recordar lo "sublime" en las torres de luz diseñadas por Albert Speer para los Rallies de Nuremberg, durante el régimen nacional socialista de Alemania, junto con su sucesión interminable de individuos uniformes que parecen llegar al infinito, "tal y como sucede en la arquitectura con una larga sucesión de columnas y en la naturaleza con una avenida de árboles”. Véase Burke, op. cit., pp. 74-76. 
aparición de las Torres de Satélite? Sin duda, son parte de un programa que encierra el propósito de llevar a cabo una restauración. Este proceso puede apreciarse y evaluarse en función de su aspecto general, que parece corresponder a una circunstancia atemporal, donde la obra es capaz de remitir a las torres del pasado - como ruina - y, al mismo tiempo, a una ciudad aún por venir - a "una fantasía de rascacielos", como se llamó a las Torres en la prensa. Esto parece contener un gesto con el que una imagen del pasado, donde hipotéticamente había una sociedad orgánica y un estilo de vida más sereno, da forma a una imagen de lo que se desearía para la ciudad del futuro. ${ }^{60}$

Esta restauración se deriva del romanticismo que encierra el proyecto de arquitectura emocional. En su crítica al privilegio de la funcionalidad en la arquitectura moderna, el proyecto compartido de Goeritz y Barragán guarda un cuestionamiento inmanente a la modernidad, que encumbra a la razón y la instrumentalidad para alcanzar fines. Un estilo de vida sereno, una sociedad orgánica, la noción de lo espiritual y la reflexión introspectiva son algunos de los valores e ideales tal vez precapitalistas y premodernos que la arquitectura emocional intentaba recuperar en el marco de su crítica latente. ${ }^{61}$ Como se sabe, el romanticismo reviste un carácter contradictorio: "su naturaleza es coincidentia oppositorium" y por ello resulta, simultáneamente, retrógrado y utópico, revolucionario y contrarrevolucionario. ${ }^{62}$ Por lo tanto, puede haber distintos tipos de romanticismos, con sus respectivas tendencias, desde uno de corte próximo al fascismo hasta otro de carácter revolucionario y utópico. Se puede decir que, según el tipo de romanticismo, se definirán las características de la restauración. ¿Cómo funciona esto en el proyecto de la arquitectura emocional? ¿Qué tipo de romanticismo lo motiva? ¿Qué clase de restauración promueve? Sin duda, éste no es el espacio para desarrollar en profundidad una serie de cuestiones y las Torres de Satélite no son prueba suficiente para llegar a conclusiones definitivas al respecto. Sin embargo, sí pueden plantearse primeras aproximaciones. Tal como se puede ver en esas Torres, y en general en el

6o. El regreso a una sociedad orgánica y la articulación de sitios propicios para un estilo de vida más sereno dentro del caótico mundo moderno representan ideales de los proyectos de Goeritz y Barragán, respectivamente. La búsqueda de una sociedad orgánica tiene que entenderse como la intención de materializar un ideal que, quizá, nunca llegó a existir.

6I. Michael Löwy y Robert Sayre, Romanticism Against the Tide of Modernity, Durham, Duke University Press, 200I, pp. I7-I9 (originalmente publicado como Révolte et mélancolie: Le romantisme à contre-courrant de la modernité, 1992).

62. Ibidem, p. I. 
6. Proyecto para la Plaza Norte de Ciudad Satélite. Imagen del medio en la segunda columna, tomada de "Pani, México. Un problema, una solución”, op. cit. (supra n. 8), s.p. Fondo Mathías Goeritz. Archivo Cenidiap/InBA.

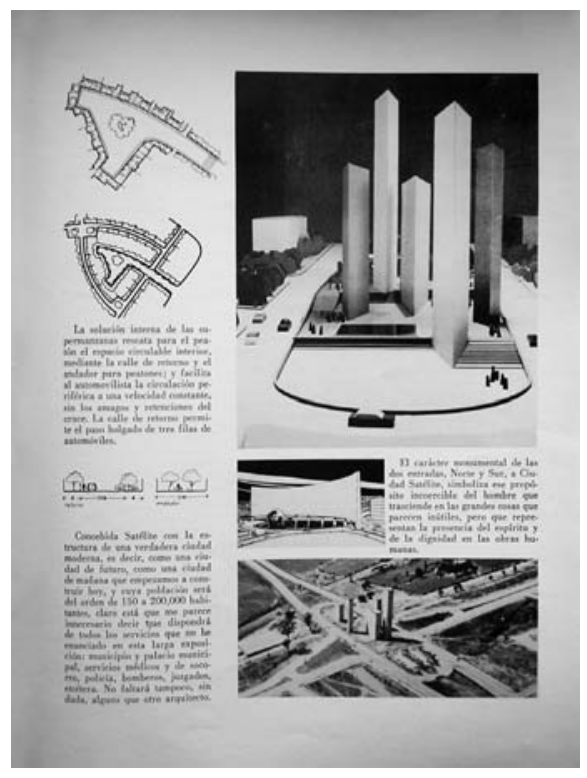

proyecto urbano del que formaban parte, si hay un tipo de romanticismo entonces es de carácter conservador. En el proyecto original de Ciudad Satélite, a pesar de su modernidad, se aprecia la intención de restaurar un tipo de arquitectura que aspira a relacionarse con la categoría de lo sublime, intenta provocar una emoción y pretende volver a ser una expresión comunicativa. También se encuentra el ideal de crear una sociedad que nazca de la convivencia, una comunidad idílica donde la razón que impera es comunicativa. La crítica a la modernidad - a su disolución de los lazos sociales, a su desencantamiento del mundo, a su mecanización y racionalización- que encierra dicho proyecto, sin embargo, no busca trastocar el estado de las cosas imperante en la sociedad ni tampoco sus formas de gobierno ni su lógica y su distribución sociales. De hecho, se podría decir que los refuerza. De aquí su franco conservadurismo.

\section{Las Torres como una ruina en el presente}

Muy poco tiempo después de que finalizó la construcción de las Torres, el proyecto de Ciudad Satélite se desechó. Así, el asentamiento dejó de ser una ciudad pla- 
nificada y, por lo tanto, no hubo necesidad de construir otra obra monumental para terminar de marcar sus límites, mucho menos algo semejante a una muralla. El centro comercial nunca apareció en la forma que originalmente se le atribuyó y nunca hubo otra iniciativa de continuar la propuesta de Pani para construir una red de ciudades satélite. Al comparar el ideal propuesto con la condición presente - caracterizada por una desbordada mancha urbana con todas las problemáticas que implica-, la pregunta que surge es ¿qué fue lo que pasó? La respuesta puede hallarse si se piensa en el fracaso del proyecto de Ciudad Satélite a resultas de otro plan mayor que también fracasó: en breve, el de los ideales del proyecto nacional abanderado por una serie de gobiernos capitalinos, estatales y federales a lo largo de más de cinco décadas. La visibilidad del conjunto, así como su efecto de elevación y creciente verticalidad, ha sido trastocada desde que se instaló un puente peatonal a finales de los años sesenta y, hoy, se encuentra en riesgo como nunca por el proyecto del Segundo Piso del Bicentenario, un viaducto elevado de peaje con una extensión de 22 kilómetros que pasaría justo a sus lados. ${ }^{63}$ Hoy en día, las Torres viven un proceso de franco deterioro, su paisaje original ha sido sepultado por un caos constructivo y su plataforma ha sido reducida por las arterias de circulación vehicular. Junto al sistema vial, este conjunto escultórico permanece como un vestigio de lo que se pensó en el proyecto original de la nueva ciudad. Las Torres de Satélite son hoy una ruina monumental que anuncia un futuro anhelado y planeado que, sin embargo, nunca se concretó. \$े

63. Sobre el Segundo Piso del Bicentenario, Víctor Jiménez ha escrito un par de artículos en el periódico Reforma, en la sección "Arquitextos": "Malas perspectivas", publicado el 20 de noviembre de 2007, y "Arte urbano y política", el I de enero de 2008. 